\title{
Exploring Frontline Healthcare Worker's Stress and Recovery Off-Shift during the COVID-19 Pandemic
}

\author{
Hoora Emami \\ Dalla Lana School of Public Health, University of Toronto, Toronto, Canada \\ E-mail: hoora.emami@mail.utoronto.ca
}

Received October 28, 2020

Accepted for publication December 23, 2020

Published online September 26, 2021

\begin{abstract}
I completed my practicum with 4 YouandMe, a non-profit created to aid individuals who are interested in sharing healthrelated data using smartphones and other wearable devices so that they can better understand and navigate health conditions. The Stress and Recovery Study used the Oura ring and smartphones to track and understand the multidimensional components of stress and recovery off-shift in frontline healthcare workers during the current COVID-19 pandemic. My role in this study was actively working as a clinical research coordinator and digital participant engagement expert. This role consisted of calling participants and asking them about their overall study experience, details regarding their stress triggers, their home and work environments, and use of their Oura ring. I was responsible for maintaining contact with about 70 participants and creating contact logs after each phone call. The purpose of these phone calls is to provide support and encourage participant adherence to the study tasks. In addition to this primary role, I also completed an emerging COVID-19 hotspot map that was used in the recruitment process of the study. I outlined regions in the U.S that may become hotspots for COVID cases and may subsequently translate to a higher stressed group of healthcare workers in those areas. Additionally, I contributed to developing adherence tracking frameworks and other study materials used by team members. This study is contributing to the public health literature by using novel methodologies including digital approaches to understanding stress. Looking at digital stress responses and biometric data as signals to predict infection may inform other tools to aid in early detection. Finally, the study aims to determine whether resiliency factors and some social determinants of health modify stress and recovery.
\end{abstract}

